Cultures \& Conflits

45 | printemps 2002

De Tampere à Séville : bilan de la sécurité européenne $(1 / 2)$

\title{
Convention Europol : Office européen de police
}

\section{Union européenne}

\section{(2) OpenEdition \\ Journals}

Édition électronique

URL : http://journals.openedition.org/conflits/789

DOI : 10.4000/conflits.789

ISSN : $1777-5345$

Éditeur :

CCLS - Centre d'études sur les conflits lilberté et sécurité, L'Harmattan

Édition imprimée

Date de publication : 1 mars 2002

ISBN : 2-7475-3029-9

ISSN : 1157-996X

\section{Référence électronique}

Union européenne, «Convention Europol: Office européen de police », Cultures \& Conflits [En ligne], 45 | printemps 2002, mis en ligne le 22 mars 2006, consulté le 30 mars 2021. URL : http:// journals.openedition.org/conflits/789; DOI : https://doi.org/10.4000/conflits.789

Ce document a été généré automatiquement le 30 mars 2021.

Creative Commons License 


\title{
Convention Europol : Office européen de police
}

\author{
Union européenne
}

\section{1) OBJECTIF}

2 Améliorer la coopération policière entre les Etats membres pour lutter contre le terrorisme, le trafic illicite de drogues et les autres formes graves de la criminalité internationale.

3 2) MESURE DE L'UNION

4 Acte du Conseil, du 26 juillet 1995, portant établissement de la convention portant création d'un Office européen de police (convention Europol).

3) CONTENU

6 1. Cette convention vise à mettre en place un office européen de police dénommé «Europol », implanté aux Pays-Bas, à La Haye. Il a pour mission d'améliorer l'efficacité des services compétents des Etats membres et leur coopération dans des domaines de plus en plus nombreux : la prévention et la lutte contre le terrorisme ;

8 le trafic illicite de stupéfiants ;

9 le trafic des êtres humains ;

10 les filières d'immigration clandestine ;

11 le trafic illicite de matières radioactives et nucléaires ;

12 le trafic illicite de véhicules ;

13 la lutte contre le faux monnayage de l'euro ;

14 et le blanchiment d'argent lié aux activités criminelles internationales.

15 2. Europol remplit en priorité les fonctions suivantes:

16 faciliter l'échange d'informations entre les Etats membres;

17 rassembler et analyser les informations et renseignements; 
communiquer aux services compétents des Etats membres les informations les concernant et les informer immédiatement des liens constatés entre des faits délictueux :

faciliter les enquêtes dans les Etats membres ;

gérer des recueils d'informations informatisés.

3. Chaque Etat membre crée ou désigne une unité nationale chargée d'exécuter les fonctions énumérées ci-dessus. L'unité nationale est le seul organe de liaison entre Europol et les services nationaux compétents. Elle envoie auprès d'Europol au moins un officier de liaison, chargé de représenter les intérêts de celle-ci au sein d'Europol.

4. En vue de remplir ses fonctions, Europol gère un système d'informations informatisé. Alimenté directement par les Etats membres, le système d'informations est directement accessible, en consultation, aux unités nationales, aux officiers de liaison, aux directeur et directeurs adjoints, ainsi qu'aux agents d'Europol dûment habilités.

5. Outre des données à caractère non personnel, peuvent également figurer dans ce système d'informations des données à caractère personnel. Tout fichier automatisé de données à caractère personnel, doit faire l'objet, de la part d'Europol, d'une instruction de création soumise à l'approbation du conseil d'administration. Les données à caractère personnel extraites du système d'informations ne peuvent être transmises ou utilisées que par les services compétents des États membres pour prévenir et lutter contre la criminalité relevant de la compétence d'Europol, et contre les autres formes graves de criminalité.

6. Toute personne désirant accéder aux données la concernant, stockées à Europol, peut formuler gratuitement une demande dans tout Etat membre de son choix à l'autorité nationale compétente, qui saisit alors Europol et avise le requérant qu'Europol lui répondra directement. Toute personne est en droit de demander à Europol de rectifier ou d'effacer des données erronées la concernant.

7. Une autorité de contrôle commune indépendante est chargée de surveiller l'activité d'Europol afin de s'assurer que le stockage, le traitement et l'utilisation des données dont disposent les services d'Europol ne portent pas atteinte aux droits des personnes.

8. Les organes d'Europol sont :

Le conseil d'administration, composé d'un représentant de chaque Etat membre. La présidence du conseil d'administration est assurée par le représentant de l'Etat membre qui exerce la présidence du Conseil.

Le directeur, nommé par le Conseil, pour une période de quatre ans, renouvelable une fois. Le directeur et les directeurs adjoints peuvent être révoqués après avis du conseil d'administration.

Le contrôleur financier, nommé à l'unanimité par le conseil d'administration et responsable devant lui.

Le comité budgétaire, composé d'un représentant de chaque Etat membre.

9. Le budget est financé par les contributions des Etats membres et par d'autres recettes occasionnelles. Les comptes concernant toutes les recettes et dépenses inscrites au budget, ainsi que le bilan des éléments actifs et passifs d'Europol sont soumis à un contrôle annuel. Le contrôle des comptes est effectué par un comité de contrôle, composé de trois membres désignés par la Cour des comptes des Communautés européennes. 

lequel interviennent des données entachées d'erreurs de droit ou de fait, stockées ou traitées à Europol. Seul l'Etat membre où le fait dommageable s'est produit peut faire l'objet d'une action en indemnisation de la part de la victime.

Suite à l'entrée en vigueur de la convention, différentes mesures ont été prises pour permettre la mise en place de l'office européen de police. Elles concernent les droits et obligations des officiers de liaison, les règles d'application pour les fichiers, le règlement intérieur de l'autorité de contrôle commune, le statut du personnel, la réglementation en matière de protection du secret, le règlement financier l'accord de siège, le protocole sur les privilèges et immunités et les accords sur les privilèges et immunités des officiers de liaison. Ainsi, Europol a pu entamer ses activités le 1er juillet 1999, date à laquelle il a remplacé l'unité «Drogues» Europol (UDE), créée provisoirement en 1995. membre de l'Union européenne.

4) ÉCHÉANCE FIXÉE POUR LA MISE EN OEUVRE DE LA LÉGISLATION DANS LES ÉTATS MEMBRES

Non requis.

5) DATE D’ENTRÉE EN VIGUEUR (si elle ne concorde pas avec la date précédente)

01.10.1998

6) RÉFÉRENCES

Journal officiel C 316, 27.11.1995

7) TRAVAUX ULTÉRIEURS

Le 23 juillet 1996, le Conseil a adopté un acte établissant, sur la base de l'article K.3 du traité de l'Union européenne, le protocole concernant l'interprétation, à titre préjudiciel, par la Cour de justice des Communautés européennes de la convention portant création d'un Office européen de police [Journal officiel C 299, 09.10.1996].

Les Etats membres, par une déclaration faite au moment de la signature du protocole ou même postérieurement, peuvent accepter la compétence de la Cour de justice pour statuer, à titre préjudiciel, sur l'interprétation de la convention.

Les Etats membres, dans leur déclaration, peuvent indiquer :

soit que toute juridiction dont les décisions ne sont pas susceptibles de recours de droit interne est légitimée à demander l'interprétation à titre préjudiciel de la Cour de justice sur une question soulevée devant elle et portant sur l'interprétation de la convention;

soit que toute juridiction a la faculté de demander l'interprétation d'une question soulevée devant elle.

4 Le 29 avril 1999, le Conseil a adopté une décision étendant le mandat d'Europol à la lutte contre le faux monnayage et la falsification des moyens de paiement [Journal officiel C 149, 28.05.1999].

La décision précise que les termes «faux monnayage » et « falsification des moyens de paiement » doivent être entendus au sens de l'article 3 de la convention de Genève du 20 avril 1929 pour la répression du faux monnayage, qui s'applique à la fois aux liquidités et à d'autres moyens de paiement. 
49 Le mandat d'Europol s'étendra à ces délits à partir de la date de démarrage de ses activités.

Le 30 novembre 2000, le Conseil a adopté l'acte établissant, sur la base de l'article 43 , paragraphe 1, de la convention portant création d'un office européen de police (convention Europol), le protocole modifiant l'article 2 et l'annexe de ladite convention [Journal officiel C 358, 13.12.2000].

51 Cet acte étend la compétence d'Europol au blanchiment de l'argent en général, quel que soit le type d'infraction à l'origine des produits blanchis.

Le 17 octobre 2000, le Conseil a adopté une décision portant création d'un secrétariat pour les autorités de contrôle communes chargées de la protection des données, instituées par la convention portant création d'un office européen de police (convention Europol), la convention sur l'emploi de l'informatique dans le domaine des douanes et la convention d'application de l'accord de Schengen relatif à la suppression graduelle des contrôles aux frontières communes (convention de Schengen) [2000/641/ JAI - Journal officiel L 271, 24.10.2000].

53 Le 30 novembre 2000, le Conseil a adopté une recommandation aux Etats membres concernant l'appui d'Europol aux équipes communes d'enquête instituées par les Etats membres.

54 Dans ce document, le Conseil décrit les façons dont Europol peut apporter son appui aux équipes communes d'enquête et recommande aux Etats membres d'utiliser pleinement ces possibilités. (...)

\section{INDEX}

Mots-clés : construction européenne, coopération internationale, droit européen, police, textes et documents officiels 